\section{DOS REALIZACIONES PREFABRICADAS EN BOLONIA/ITALIA}

832-39

\section{SINOPSIS}

Se describen dos obras realizadas mediante elementos prefabricados en Bolonia (Italia) por la empresa Grandi Lavori. Se trata de un conjunto de tres grandes torres con proyecto del profesor Kenzo Tange y una construcción-piloto en el recinto ferial.

La reciente visita al SAIE'81 Salón Internacional de la Industrialización de la Edificación en Bolonia, nos ha permitido contemplar dos importantes realizaciones prefabricadas de muy distinta entidad, pero que tratamos conjuntamente por tener nexos comunes: ejecutadas por la misma empresa, Grandi Lavori; ubicadas en el distrito ferial de Bolonia y realizadas utilizando técnicas de prefabricación. Nos referimos al complejo en construcción conocido como "Distrito Ferial" y a un edificio-piloto en el interior del recinto de exposiciones.

\section{El Complejo "Distrito Ferial»}

Se trata sin duda de una importante realización por el volumen de obra, por la calidad de su ejecución y por los medios tecnológicos empleados. Según datos de la empresa constructora, la operación supone unos $700.000 \mathrm{~m}^{3}$ de construcción mayoritariamente realizados mediante elementos prefabricados que tipológicamente se apartan bastante de lo realizado hasta el presente (Fig. 1).

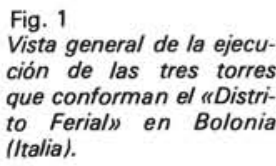
ción de las tres torres que conforman el "Distrito Ferial's en Bolonia (Italia).

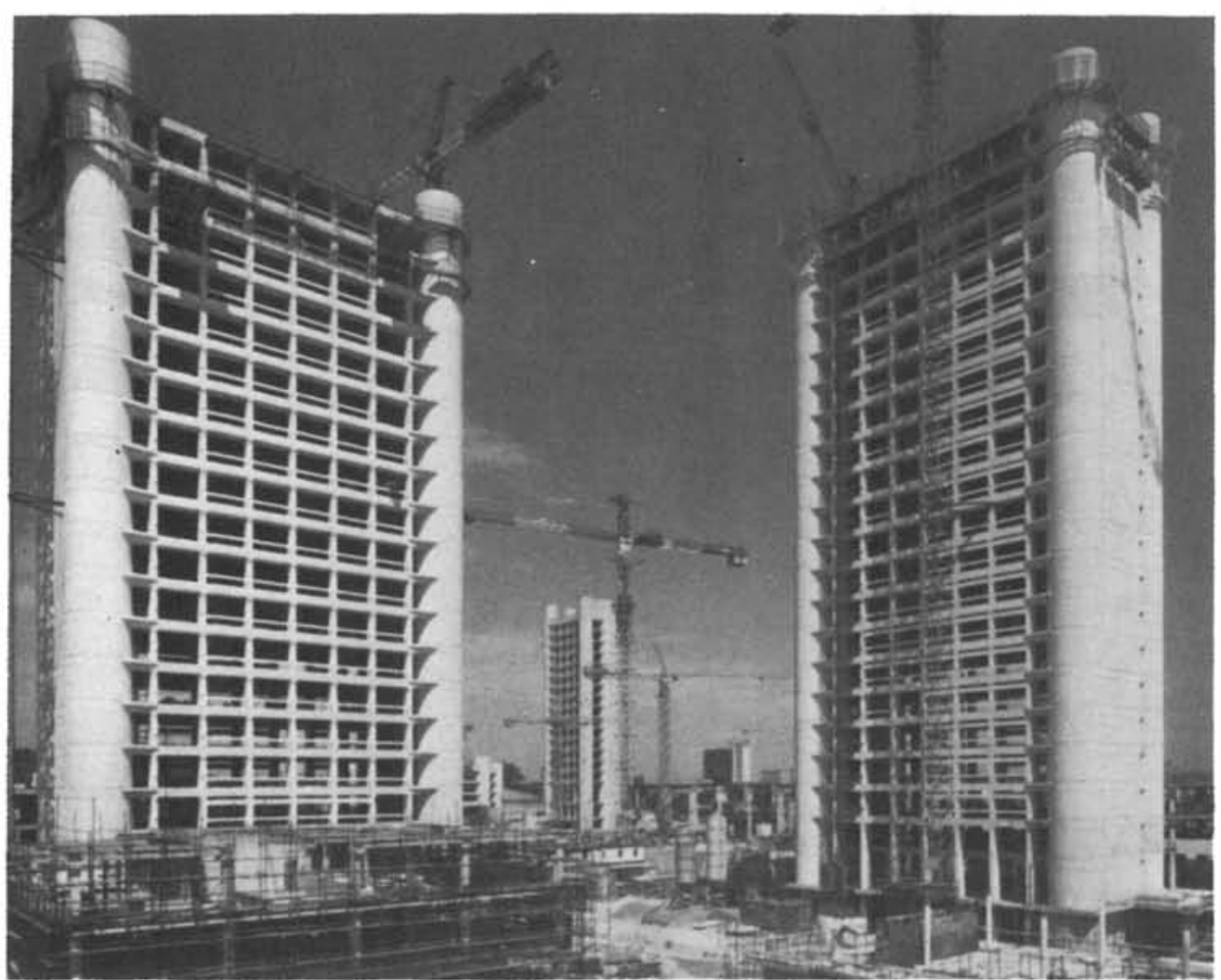


En el esquema adjunto (Fig. 2), se muestra la forma fundamental de trabajo y la descomposición en elementos básicos de cada una de las tres torres de 21 plantas.

Uno de los elementos fundamentales y sin duda el más característico de este conjunto es el hueco de escaleras. Se trata de grandes cilindros de $5 \mathrm{~m}$ de diámetro por $3,64 \mathrm{~m}$ de altura y $8 \mathrm{~cm}$ de espesor de pared, que se prefabrican en una pieza repitiéndose este elemento 252 veces en el conjunto de las tres torres (Figs. 3 y 4 ). También son prefabricados los cajones de ascensores y montacargas (378 elementos). Las losas nervadas de forjados de grandes luces permiten una total libertad de distribución en planta; el número de losas en « $\pi$ ) de $14 \mathrm{~m}$ de luz es de 880 , que coincide igualmente con el de pilares-jácena igualmente prefa-

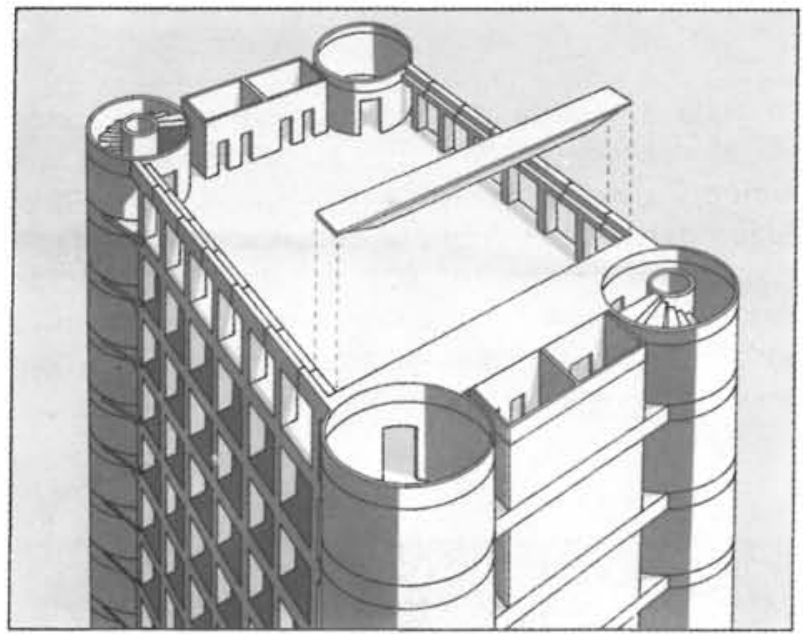

Fig. 2

Esquema de la descomposición en elementos.

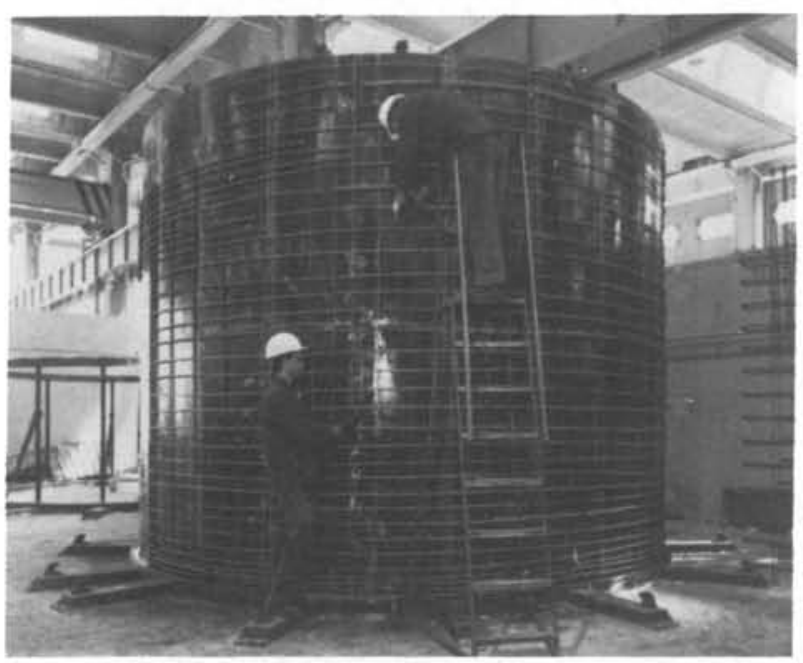

Fig. 3

Preparación en taller de la armadura de los cilindros de cajas de escaleras.

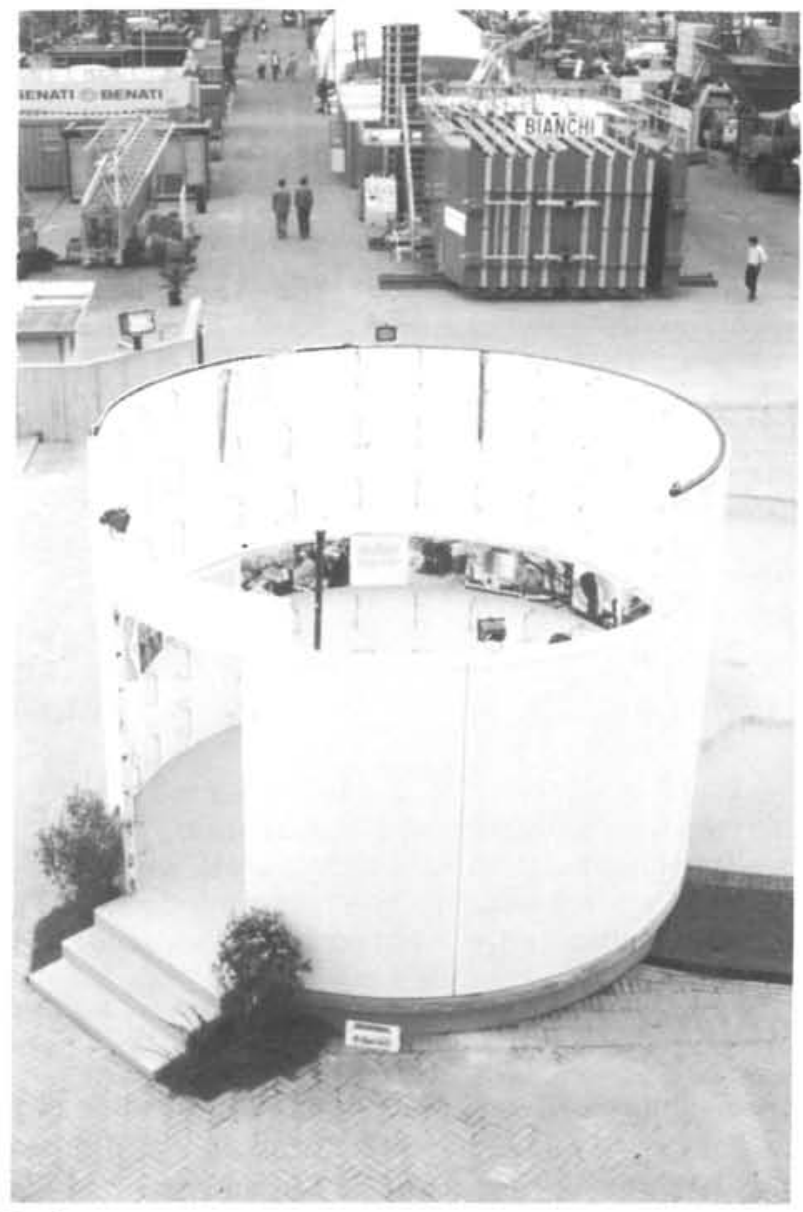

Fig. 4

Elemento de caja de escalera exhibido en el SAIE'81.

bricados y que presentan forma de "T" sobre cuyas alas apoyan sendas losas nervadas de forjado.

La estereotomia de la obra es espectacular y al mismo tiempo sencillisima, lo que le da un aspecto de sinceridad global y claridad estructural. Sin lugar a dudas denota esta obra la notable influencia de un buen proyecto - en este caso obra del maestro Kenzo Tange- y de la adecuación de la tecnologia empleada sobre el resultado de la obra (Fig. 5).

Como ya se ha dicho, toda la estructura es prefabricada a base de elementos con cemento puzolánico blanco, para cuya producción se han empleado moldes metálicos con vibradores fijos y sistemas de mando oleodinámicos. La factoria de producción Pontuhio-Marconi se encuentra a 18 kilómetros de Bolonia. Cuenta la obra con un parque de acopio donde se han tomado toda clase de precauciones para evitar el deterioro de los paramentos vistos.

Para el montaje se utilizan grúas-torre relativamente poco importantes $(20 \mathrm{t} \cdot \mathrm{m}$ de capacidad $)$ ya que 


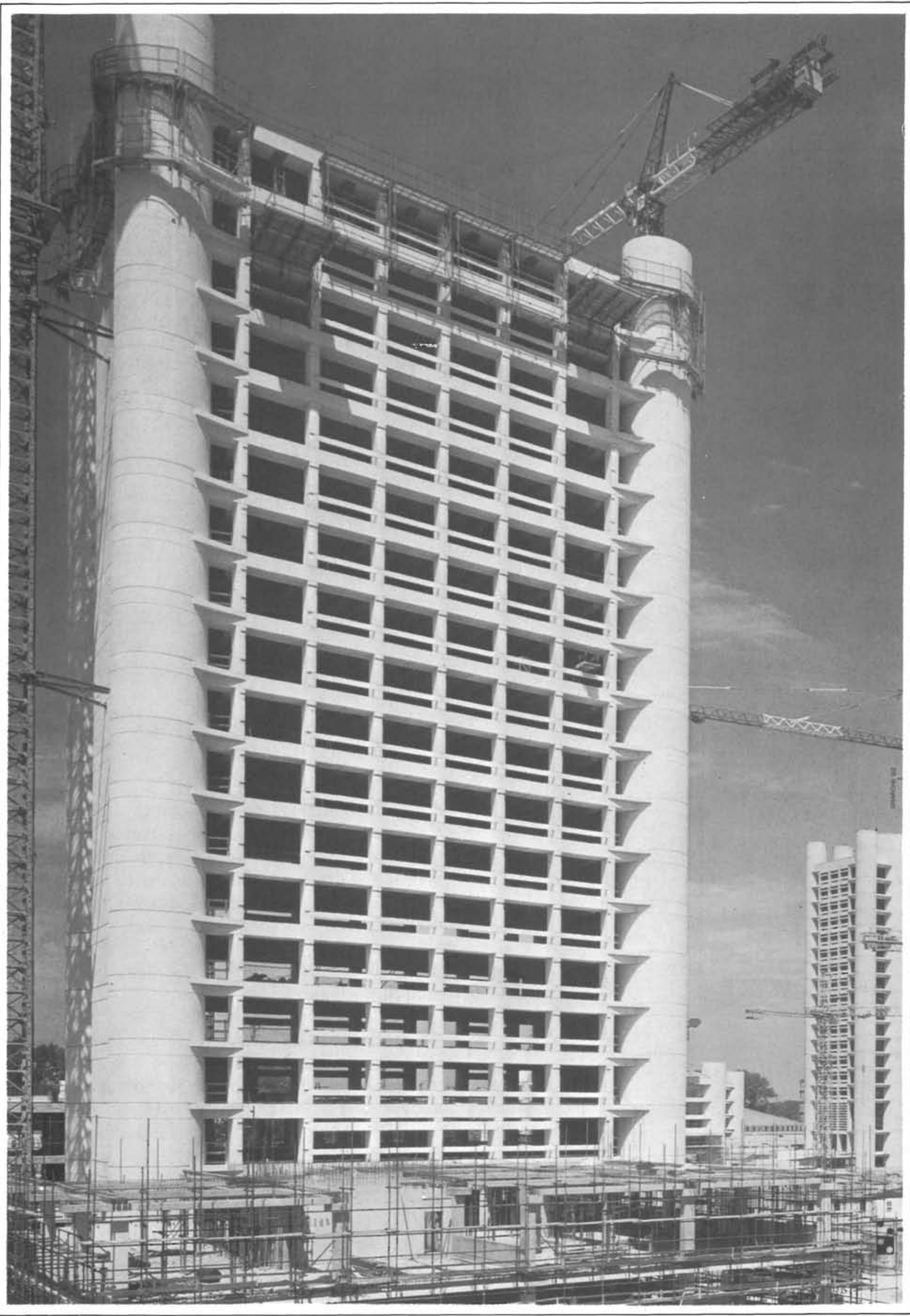




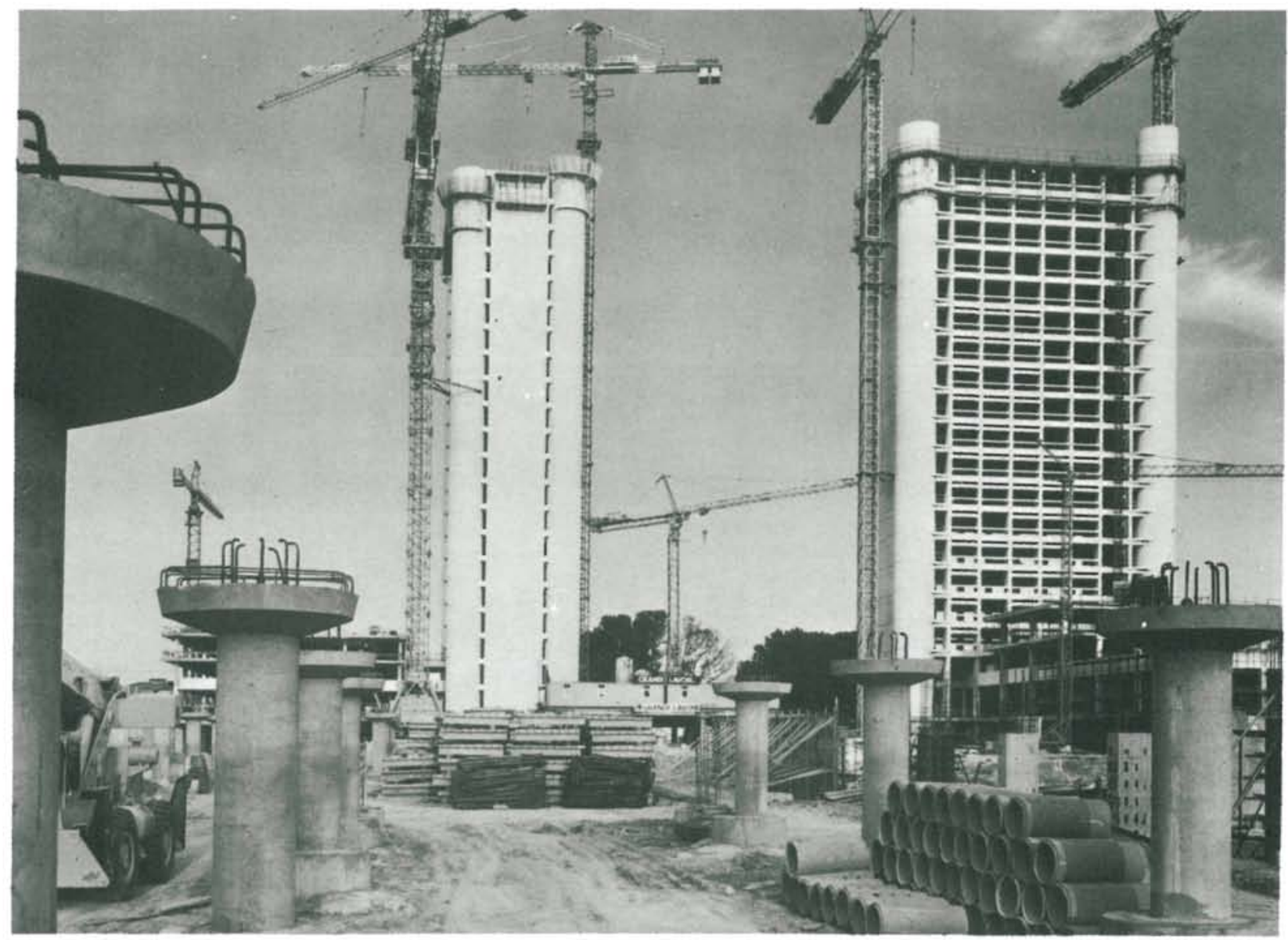

Fig. 6

Vista general de la obra y detalle del arriostramiento de las grüas-torre en el edificio.

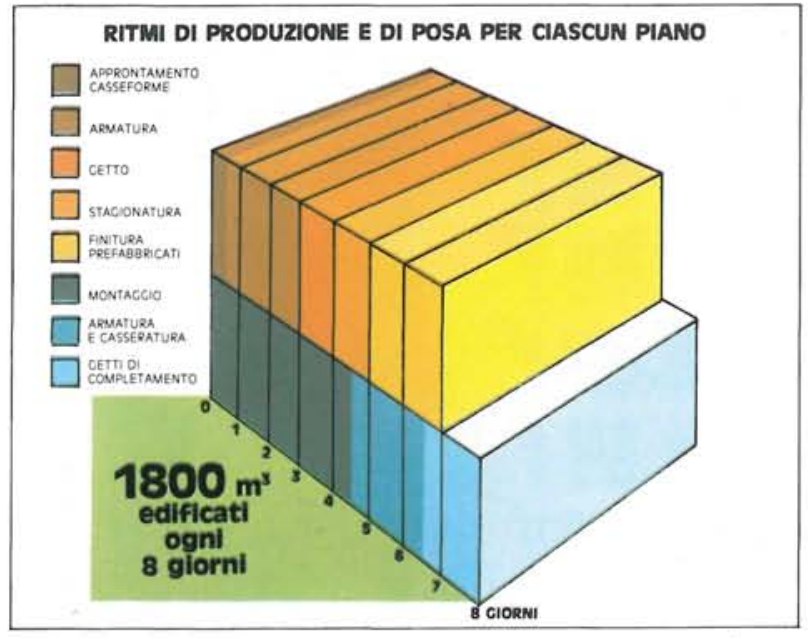

se ha estudiado cuidadosamente su ubicación y se han arriostrado a la propia estructura del edificio en construcción (Fig. 6). La secuencia de montaje es: cilindros de escaleras; tramos helicoidales de escalera; cajas de ascensores y montacargas; elementos pilares-jácena y losas nervadas en $« \pi »$,
Colocados los cuatro cilindros o cajas de escaleras en las esquinas de cada planta y montados en el interior de los mismos los tramos de escalera helicoidales, que tienen altura de una planta, se procede al hormigonado de una capa de $17 \mathrm{~cm}$ de espesor que junto a los $8 \mathrm{~cm}$ de los cilindros prefabricados dan el total de $25 \mathrm{~cm}$. Como se puede apreciar en la Fig. 4, los cercos de espera en los cilindros prefabricados facilitan la solidarización al hormigonar.

Posteriormente se montan los elementos en "T". elementos pilar-jácenas y sobre las alas de los mismos, que cuentan con los debidos rebajes, apoyan sendas losas nervadas de sección en « $\pi$; una vez colocados todos los elementos de la planta se procede al hormigonado de la viga de atado perimetral y de las juntas longitudinales entre losas de forjado.

La figura 7 recoge un esquema de los tiempos empleados en las distintas fases. El ritmo de montaje es de una planta, equivalente a $1.800 \mathrm{~m}^{3}$ construidos, cada ocho dias de trabajo. 
Informes de la Construcción/329

\section{Edificio-exhibición}

Aunque reducida en dimensiones, ya que se trata de una realización piloto expresamente construida para su exhibición en la feria, nos ocupamos de este edificio por la calidad de su ejecución que presenta no pocas conexiones con la obra anteriormente descrita. (Figs. 8, 9 y 10).
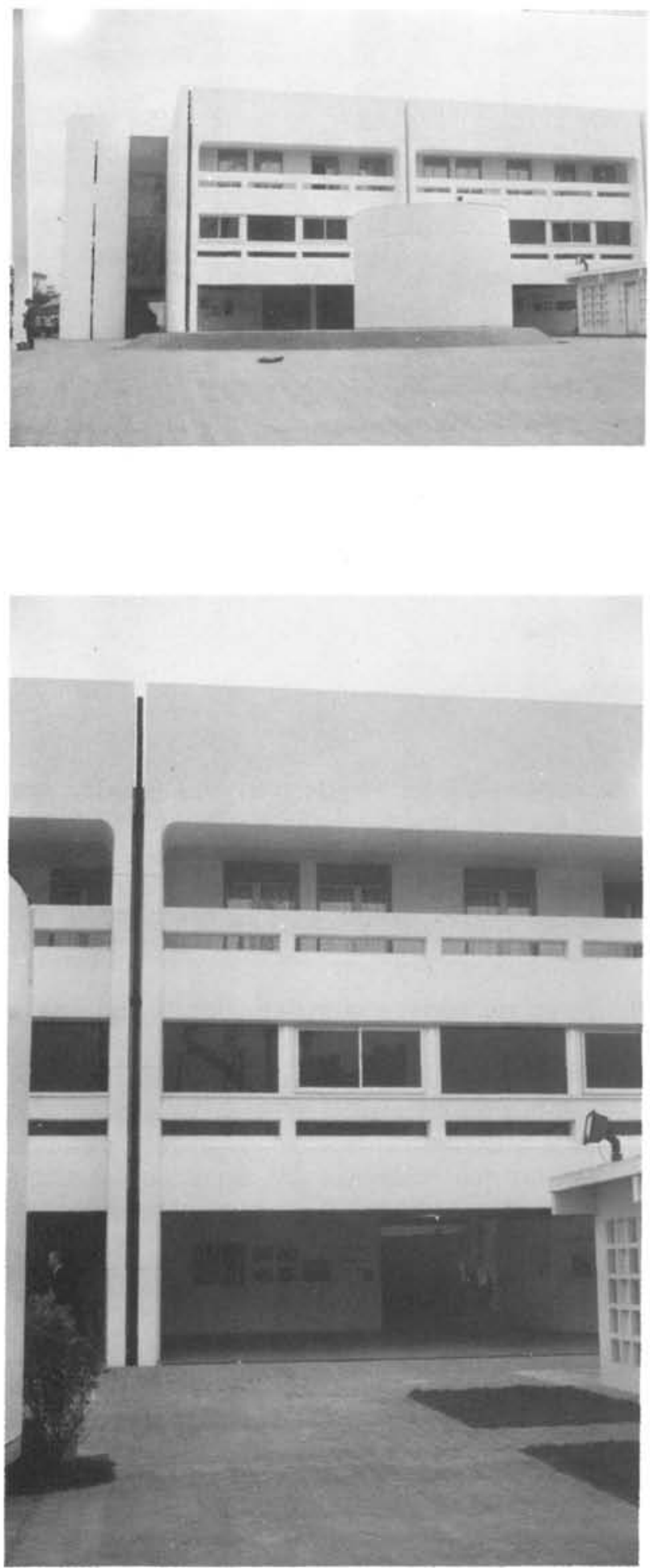

Figs. 8 y 9

Alzado frontal del edificio-piloto exhibido en el SAIE'81.

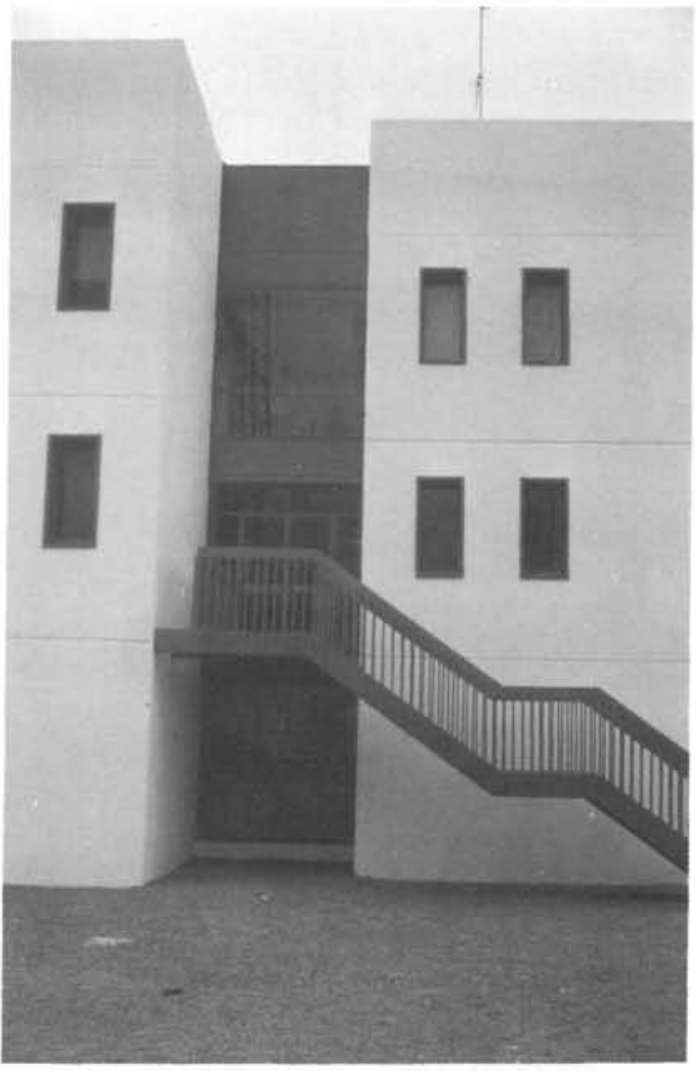

Fig. 10

Alzado del piñón con acristalamiento de pasillos y muestra de escalera de seguridad.

Fig. 11

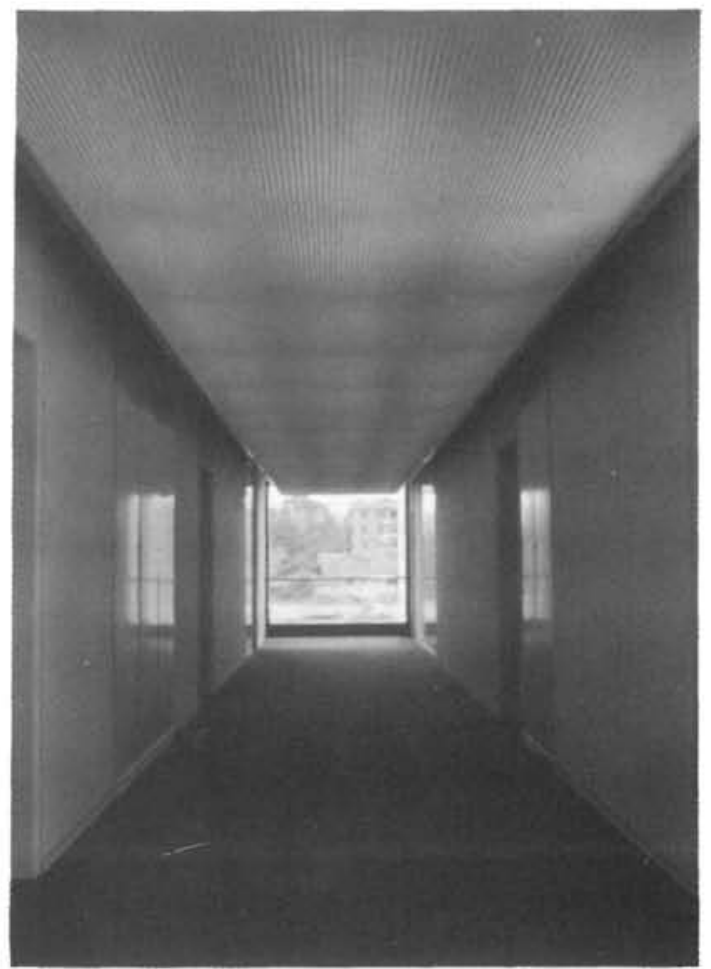




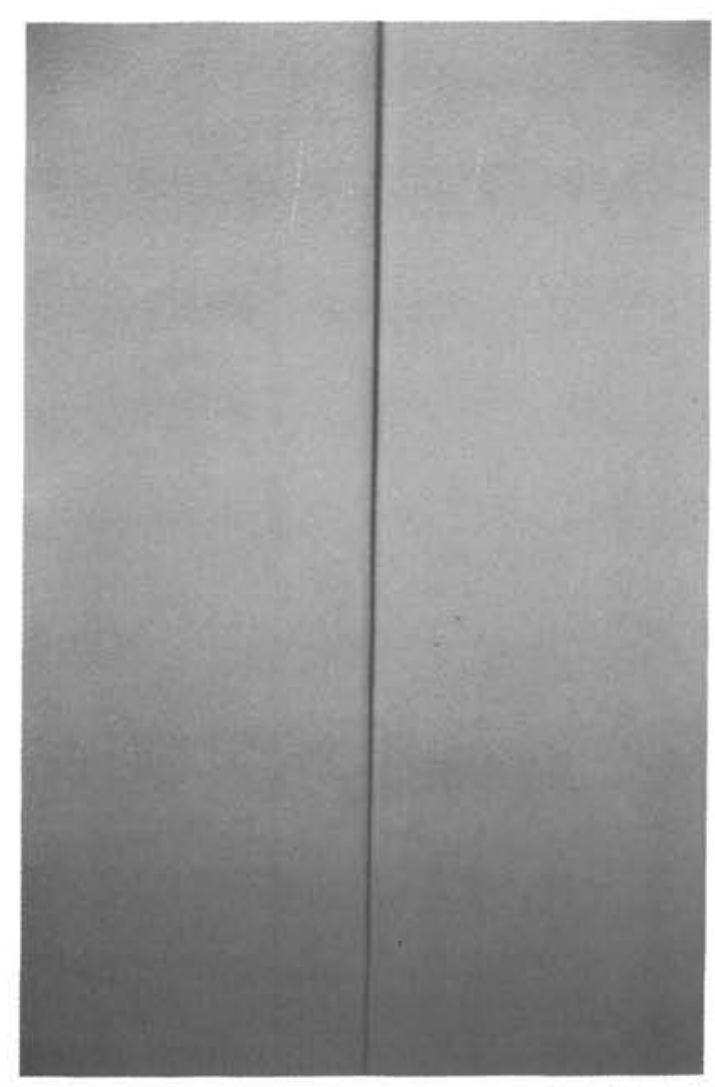

Fig. 12

Detalle de la junta longitudinal entre losas de forjado vista.

Fig. 13

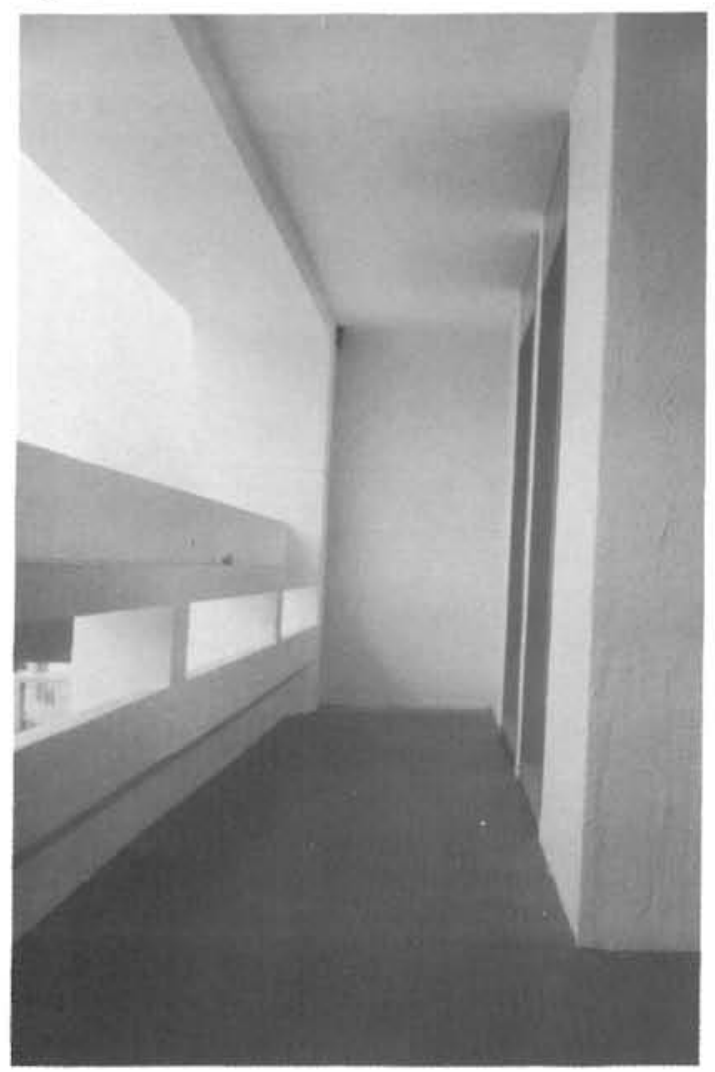

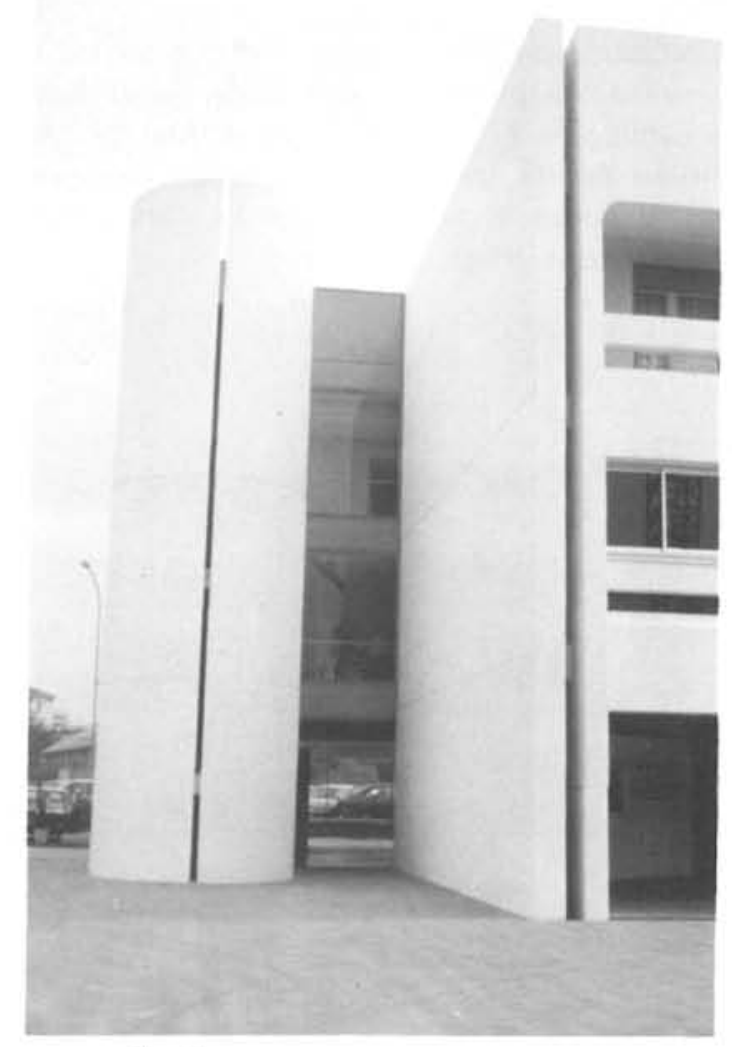

Fig. 14

Este edificio-piloto, totalmente prefabricado, responde a un "modelo" bastante experimentado por Grandi Lavori para bloques de viviendas en Italia, que en la actualidad se está empleando en la ejecución de conjuntos residenciales en algunos paises africanos.

El núcleo de edificio expuesto consta de baja y dos alturas con cuatro viviendas por planta, a las que se accede por un amplísimo pasillo (Fig. 11). La distribución en planta es libre, ya que se trata de vanos absolutamente diáfanos a base de losas de gran luz que presentan una excelente ejecución de las juntas longitudinales entre elementos de forjado que quedan vistas. (Fig. 12).

Especial cuidado de proyecto y ejecución presentan los elementos de fachada que conforman amplias logias (Fig. 13), asi como las cajas de escalera que mediante dos semicilindros prefabricados, suficientemente separados para proporcionar iluminación al interior y tramos rectos y descansillos semicirculares en su interior, resuelven de forma muy simple y con excelentes resultados estéticos la comunicación vertical (Fig. 14). 


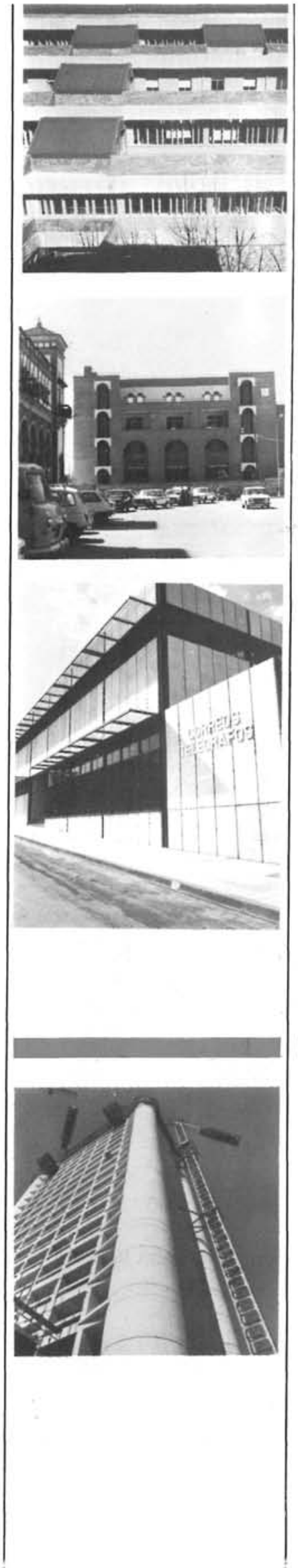

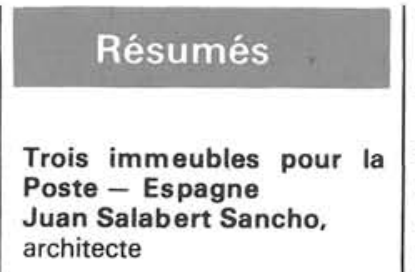

Cet article présente la construction de trois immeubles pour la Direction espagnole des Postes et Télécommunications, situés à Albacete, Jerez de la Frontera et Santa Cruz de Ténériffe.

Ces projets se caractérisent par leur caractère fonctionnel qui est donné par la distribution des es. paces, par les circulations correctes et par I'adaptation à l'environnement urbain.

Finalement, cet article décrit les principales caractéristiques de ces immeubles, la distribution des immeubles, la distribution des différents services et quelques-
uns de leurs détails constructifs les plus intéressants.

\section{.}

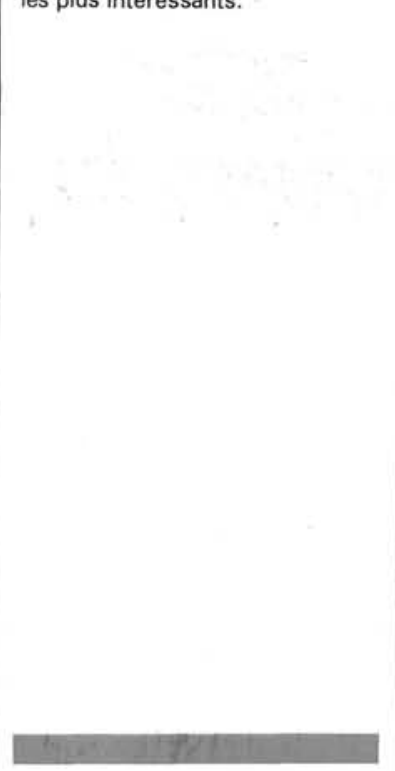

Deux réalisations préfabriquées à Bologne - Italie

On décrit deux ouvrages réalisés, à l'aide d'éléments préfabriqués, par I'entreprise Grandi Lavori, à Bologne (Italie). II s'agit donc d'un ensemble de trois grands tours suivant un projet du protours suivant un projet du pro-
fesseur Kenzo Tange et une fesseur Kenzo Tange et une
construction-pilote sur l'emplace ment de la foire.

\section{Summaries \\ Three communicatıons buildings - Spain Juan Salabert Sancho, Architect}

This article describes three communications buildings for the Central Post and Telecommunication Office, in Albacete, Jerez de la Frontera and Santa Cruz de Tenerife.

The outstanding features of these projets are their well-adapted functional nature brought about by the distribution of spaces, correct circulation and adaptation to the urban setting.

A detailed description is given of the main features of these projects, the distribution of the different services and some of their more interesting building details. ings in Bolonia - Italy

Describes two projects carried out with prefabricated elements in Bolonia (Italy) by the Grandi Lavori company. They are an ensemble of three large towers with Professor Kenzo Tange's project and a pilot building in the fairground 